\title{
Tripartite motif-containing 3 (TRIM3) enhances ER signaling and confers tamoxifen resistance in breast cancer
}

\author{
Runyi Ye $\mathbb{D}^{1,4^{凶}}$, NiJiati AiErken ${ }^{2,4}$, Xiaying Kuang ${ }^{1}$, Huijuan Zeng ${ }^{1}$, Nan Shao ${ }^{1}$, Ying Lin $^{1}$, Pian Liu (D) ${ }^{3 凶}$ and Shenming Wang $\mathbb{D}^{1 凶}$
}

(c) The Author(s) 2021

Tamoxifen resistance remains a clinical problem in estrogen receptor (ER)-positive breast cancer. SUMOylation of ERa enhances ERainduced transcription activity. Tripartite motif-containing (TRIM) proteins are a new class of SUMO E3 ligases, which regulate the SUMOylation of proteins. However, the precise molecular mechanism and function of TRIM3 in SUMOylation and the response to tamoxifen remain unclear. In the present study, we observed that TRIM3 was dramatically overexpressed in breast cancer, which correlated with tamoxifen resistance. Furthermore, TRIM3 overexpression significantly correlated with poor survival of patients with $\mathrm{ER}^{+}$breast cancer treated with tamoxifen. TRIM3 overexpression conferred cell survival and tumorigenesis, whereas knocking down of TRIM3 reduced these capabilities. Moreover, TRIM3, as a ubiquitin carrier protein 9 (UBC9) binding protein, promoted SUMO modification of estrogen receptor 1 (ESR1) and activated the ER pathway. Silencing UBC9 abolished the function of TRIM3 in regulating tamoxifen resistance. These results suggest TRIM3 as a novel biomarker for breast cancer therapy, indicating that inhibiting TRIM3 combined with tamoxifen might provide a potential treatment for breast cancer.

Oncogenesis (2021)10:60; https://doi.org/10.1038/s41389-021-00350-x

\section{INTRODUCTION}

Representing almost $25 \%$ of cancer cases among women, breast cancer remains a global challenge worldwide [1]. Estrogen receptor alpha (ERa) is the main molecular target for endocrine therapies, which antagonize the ER and suppress estrogen synthesis to inhibit tumor growth [2]. Tamoxifen, as an adjuvant endocrine therapy, is widely used as an ER antagonist in breast cancer, which blocks the binding between estrogen and the ER, and suppresses ERa target genes [3]. Unfortunately, $\sim 30-40 \%$ of patients with $\mathrm{ER}^{+}$breast cancer fail to respond (de novo resistance) or become resistant (acquired resistance) to tamoxifen, with a deadly outcome, which presents a major clinical challenge in the treatment of breast cancer $[4,5]$. Therefore, it is important to determine the underlying molecular mechanism of endocrine resistance and to develop novel potential therapies for breast cancer.

Evidence has proved that several mechanisms contribute to tamoxifen resistance, such as activation of receptor tyrosine kinases (RTK) signal transduction pathways, activation of oncogenic signaling pathways (e.g., the phosphatidylinositol-4,5-bisphosphate 3-kinase (PI3K)/protein kinase B (Akt)/mammalian target of rapamycin (mTOR) pathway, and the nuclear factor kappa B (NF-kB) pathway) and modulation of ER signaling [6-9]. The ER is widely expressed in breast cancer (almost 70\%), which is a strong predictor for tamoxifen therapy [10]. Notably, loss of estrogen receptor 1 (ESR1) expression confers tamoxifen resistance, which is reported in $15-20 \%$ of breast cancer cases $[10,11]$. However, the ER in most tamoxifen-resistant cases remains to be expressed and activated [10], suggesting that there might other mechanisms that regulate ESR1 to confer tamoxifen resistance. Emerging evidence shows that ESR1 expression is regulated by diverse aspects, including histone modification [12], DNA methylation [13], somatic mutation [10], ESR1 fusion genes [14], and post-translational modifications (PTMs) [15]. SUMOylation, as an important PTM, effects subcellular localization, protein-protein interaction, protein stability, and transcriptional activity, and is regulated by three important enzymes: Activating enzyme (E1), conjugating enzyme (E2), and ligases (E3). Sentis et al. proved that small ubiquitin-like modifier (SUMO)-1 modifies ERa via SUMOylation, which enhances ERa-induced transcription activity via improving ERa's DNA-binding property [16]. However, the mechanism and biological function of ESR1 SUMOylation in breast cancer remain unclear.

Tripartite motif-containing (TRIM) proteins belong to the RINGtype E3 ubiquitin ligase family, which are involved cellular signaling, cell growth, and tumorigenesis [17]. Furthermore, TRIM proteins are a new class of SUMO E3 ligases, transferring SUMO to substrates $[17,18]$. TRIM27, TRIM32, and TRIM36 are well-known SUMO E3 ligases, which modify SUMOylation via binding to ubiquitin-conjugating enzyme 9 (UBC9) [17, 18]. The gene encoding TRIM3 is located on human chromosome $11 \mathrm{p} 15$, which is thought to harbor tumor suppressor genes [19-21]. However, the precise molecular mechanism and function of TRIM3 in SUMOylation and the response to tamoxifen remain unclear.

In the present study, we found that TRIM3 was significantly upregulated in tamoxifen-resistant breast cancer, and was associated with poor survival of patients with breast cancer during tamoxifen therapy. Overexpression of TRIM3 conferred estrogen-independent growth and contributed to tamoxifen resistance. Experiments revealed that TRIM3 upregulated ER

\footnotetext{
${ }^{1}$ Department of Thyroid and Breast Surgery, The First Affiliated Hospital of Sun Yat-sen University, Guangzhou, Guangdong, China. ${ }^{2}$ Department of Breast and Thyroid Surgery, The Seventh Affiliated Hospital, Sun Yat-sen University, ShenZhen 518107, China. ${ }^{3}$ Cancer Center, Union Hospital, Tongji Medical College, Huazhong University of Science and

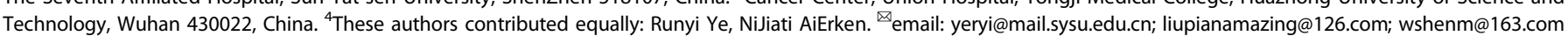


A

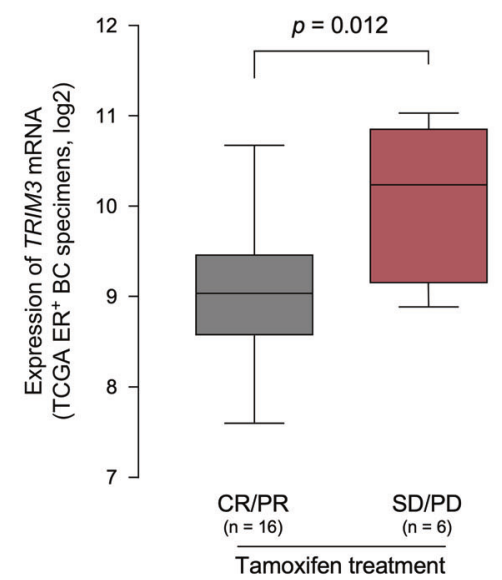

C

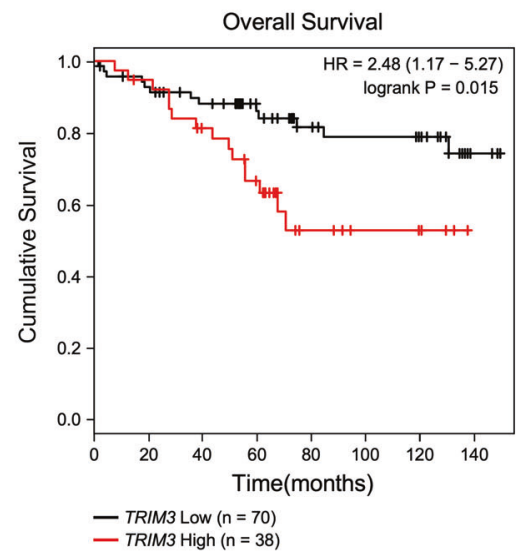

B

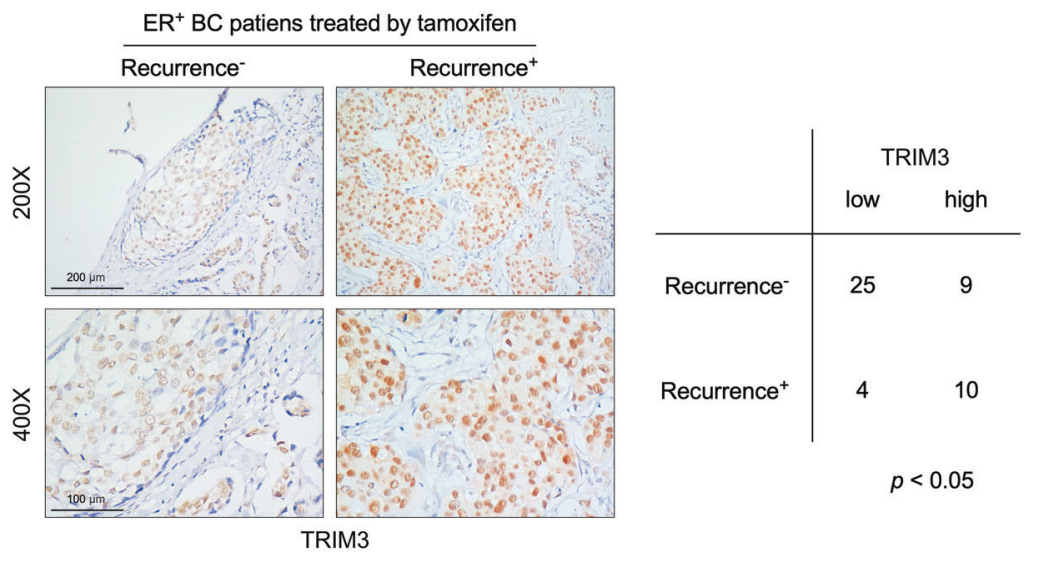

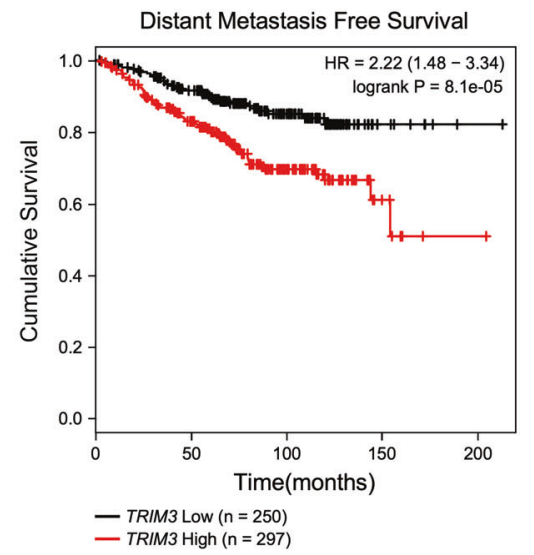

( ER positive breast cancer treated with tamoxifen only without any chemotherapy )

Fig. 1 TRIM3 overexpression promotes tamoxifen resistance in breast cancer. A A boxplot representing TRIM3 expression levels in ER ${ }^{+}$ breast cancer with tamoxifen treatment $[S D / P D$ versus $C R / P R, P=0.012$, stable disease (SD), progressive disease (PD), complete remission (CR),

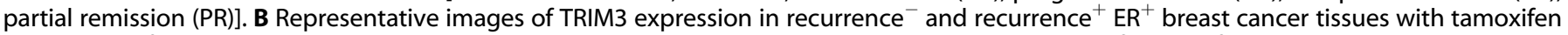
treatment (left panel). Scale bars: $200 \mu \mathrm{m}$ (upper panel) and $100 \mu \mathrm{m}$ (lower panel). The statistical significance of the TRIM3 levels is assessed in the right panel. $P<0.05$. C Analysis of the effect of TRIM3 expression on Overall Survival (OS), Relapse-Free Survival (RFS), and Distant Metastasis-Free survival (DMFS) of $\mathrm{ER}^{+}$breast cancer with tamoxifen therapy.

SUMO modification and activated the ER signaling pathway by binding to UBC9, which could be abolished by the deSUMOylation enzyme SUMO specific peptidase 1 (SENP1). Taken together, our results revealed the crucial role of TRIM3 in SUMOylation of ESR1 and the modulation of the tamoxifen response, identifying TRIM3 as a potential target to improve clinical outcomes of breast cancer.

\section{RESULTS}

\section{TRIM3 contributes tamoxifen-resistant in breast cancer}

To determine the molecular mechanism that contributes to breast cancer tamoxifen resistance, we analyzed gene expression in patients with breast cancer from TCGA database. As shown in Fig. 1A, the expression of TRIM3 was significantly higher in patients without a response to tamoxifen therapy [stable disease (SD), progressive disease (PD)] than those who responded to tamoxifen treatment [complete remission (CR), partial remission (PR)]. TRIM3 mRNA showed higher expression in $\mathrm{ER}^{+}$breast cancer than in $\mathrm{ER}^{-}$ breast cancer (Fig. S1A, $p=1.365 \mathrm{E}-68$ ). Furthermore, $48 \mathrm{ER}^{+}$clinical breast cancer specimens after tamoxifen treatment were used to examine the expression of TRIM3 protein (Table S1). As expected, TRIM3 expression was significantly higher in tamoxifen-resistant
$\mathrm{ER}^{+}$breast cancer than that in tamoxifen-sensitive $\mathrm{ER}^{+}$breast cancer (Fig. 1B). Statistical analysis revealed that the expression of TRIM3 correlated closely with cancer recurrence of patients under tamoxifen treatment (Fig. 1C). By analyzing published expression profiles obtained from breast cancer (TCGA) data, we found that levels of TRIM3 mRNA correlated positively with levels of the TRIM3 protein, and TRIM3 mRNA expression was upregulated in $\mathrm{ER}^{+}$breast cancer tissues compared with that in $\mathrm{ER}^{-}$cancer tissues and normal breast tissue (Fig. S1A, B). Consistently, Kaplan-Meier plotter showed that higher expression of TRIM3 correlated positively with poorer overall survival (OS), relapse-free survival (RFS), and distant metastasis-free survival (DMFS) in cases of $\mathrm{ER}^{+}$breast cancer with tamoxifen treatment and/or chemotherapy, but not in $\mathrm{ER}^{-}$breast cancer (Figs. 1C and S1C). These results revealed that TRIM3 might be associated with the tamoxifen resistance of $\mathrm{ER}^{+}$breast cancer.

\section{TRIM3 promotes tamoxifen resistance in $\mathrm{ER}^{+}$breast cancer in vitro}

To further determine the role of TRIM3 in regulating tamoxifen resistance, we detected the expression levels of TRIM3 in $12 \mathrm{ER}^{+}$ breast cancer cell lines. As shown in Fig. S2A, TRIM3 was highly expressed in MDA-MB-134-VI, MDA-MB-175-VII, and MDA-MB-361 
cells, and showed low expression in BT-483, CAMA-1, and MCF7 cells. Consistent with the clinical evidence, higher expression of TRIM3 in MDA-MB-134-VI, MDA-MB-175-VII, and MDA-MB-361 cells was associated with resistance to tamoxifen compared with lower expression of TRIM3 in BT-483, CAMA-1, and MCF7 (Fig. S2B, C), suggesting that TRIM3 might contribute to tamoxifen resistance in breast cancer. Furthermore, we established BT-483 and MCF7 cells that stably overexpressed TRIM3, and MDA-MB-134-VI and MDAMB-361 that were stably silenced for TRIM3 (Fig. 2A). As expected, overexpression of TRIM3-induced tamoxifen resistance and resulted in more colony formation in BT-483 and MCF7 cells, whereas silencing TRIM3 expression promoted tamoxifen sensitivity and resulted in less colony formation in MDA-MB-134-VI and MDA-MB-361 cells (Fig. 2B, C). Moreover, endogenous TRIM3 expression was upregulated in tamoxifen-resistance MCF7 cells (MCF7/TR), and silencing its expression reversed tamoxifen resistance (Fig. S3A-C). Therefore, these results revealed that TRIM3-induced tamoxifen resistance in $\mathrm{ER}^{+}$breast cancer in vitro.

\section{TRIM3 confers tamoxifen resistance in $\mathrm{ER}^{+}$breast cancer in vivo}

To further investigate the function of TRIM3 in regulating tamoxifen resistance in vivo, we first examined the anchorindependent growth ability by manipulation of TRIM3 expression in cells compared with control cells. As shown in Fig. 2D, TRIM3 overexpression promoted cell growth and the cells formed more and larger colonies compared with vector-transformed cells, whereas silencing TRIM3 expression inhibited cell growth and the cells formed fewer and smaller colonies compared with vector-transformed cells. Furthermore, we inoculated TRIM3deregulated cells subcutaneously into athymic nude mice. Consistently, TRIM3 overexpressing cells formed larger tumors compared with control vector-transformed cells (Fig. 2E). Taken together, these results revealed that TRIM3 responds to tamoxifen resistance and promotes tumorigenicity of breast cancer in vivo.

\section{TRIM3 promotes SUMOylation of ESR1 via binding to UBC9}

To further investigate the mechanism by which TRIM3 responds to tamoxifen resistance, gene set enrichment analysis was analyzed in published $\mathrm{ER}^{+}$breast cancer expression profiles from TCGA data. We found that TRIM3 levels correlated positively with ESR1 expression, suggesting that TRIM3 might be involved in regulating the ESR signaling pathway (Fig. 3A). As expected, we found that overexpression of TRIM3 increased estrogen response element (ERE)-driven luciferase reporter activity and promoted ERregulated gene expression upon tamoxifen treatment, whereas silencing of TRIM3 decreased their expression (Fig. 3B, C). However, analysis of breast cancer data in the TCGA showed that levels of TRIM3 mRNA and protein did not correlate with levels of ESR1 mRNA and protein (Fig. S4A, S5A). Furthermore, we found that E2 treatment did not induce ESR1 and TRIM3 expression in breast cancer (Fig. S4B). Overexpression of ESR1 did not affect the expression of TRIM3 (Fig. S4C). Collectively, TRIM3-induced ESR1 transcriptional activity, but did not induce ESR1 expression.

The E3 ubiquitin-protein ligase TRIM proteins are a new class of SUMO ligases (E3s), which regulate SUMOylation specificity [18]. The transcription activity of ERa is upregulated by SUMOylation via binding to UBC9, the unique SUMO E2-conjugating enzyme, and is repressed by tamoxifen treatment [16, 22-24]. Here, we found that TRIM3 overexpression increased SUMO modification of ESR1, whereas silencing TRIM3 decreased SUMOylation (Fig. 4A). Overexpression of SENP1, a deSUMOylase enzyme, decreased the SUMOylation of ESR1 and ERE luciferase reporter activity in TRIM3transduced cells compared with control cells upon tamoxifen treatment (Fig. 4B, C). Furthermore, immunoprecipitation and western blotting assays showed that TRIM3 formed a complex with ESR1 and UBC9, suggesting that TRIM3 might regulate the SUMOylation of ESR1 by binding to ESR1, UBC9, and SUMO1 activating enzyme subunit 1 (SAE1) (Fig. 4D). Silencing UBC9 in TRIM3-transduced cells reduced the SUMOylation of ESR1 and ERE luciferase reporter activity (Fig. S5B, C). To further investigate the binding of TRIM3 and ESR1, three truncated ESR1 fragments were constructed (Fig. 4E). Immunoprecipitation and far-western blotting assays showed that TRIM3 interacted with the DNA-binding fragment of ESR1 (ER2 fragment) and directly interacted with Flagtagged ESR1 cell lysate (Fig. 4F, G). Taken together, these data revealed that TRIM3 promotes SUMOylation and activates the transcription activity of ESR1 by interacting with ESR1 and UBC9.

\section{UBC9 is required for tamoxifen resistance effect of TRIM3}

To further investigate whether SUMOylation of ESR1 is required to promote the tamoxifen resistance effect of TRIM3, the effect of silencing of UBC9 and overexpressing SENP1 on TRIM3-induced tamoxifen resistance were examined. As shown in Fig. 5A, B, silencing UBC9 and overexpressing SENP1 in TRIM3-transduced cells resulted in formed reduced colony formation, which promoted tamoxifen sensitivity. Furthermore, silencing UBC9 in TRIM3-transduced cells reversed the ability of TRIM3 to induce tamoxifen resistance and decreased tumorigenicity in vivo (Fig. 5C). Consistently, the catalytic dead mutant TRIM3 (C22A/ C25A) rescued tamoxifen sensitivity in vitro and in vivo, implying that regulation of TRIM3 in tamoxifen resistance was dependent on its catalytic activity (Fig. 5A, B, Fig. S6). Taken together, these results suggest that UBC9 is required for TRIM3 regulation of ESR1 SUMOylation and the promotion of tamoxifen resistance in breast cancer.

\section{TRIM3 correlates with ESR1 SUMOylation and tamoxifen resistance in breast cancer}

To further investigate the correlation of TRIM3 and ESR1 in regulating tamoxifen resistance, 10 freshly collected clinical breast cancer samples were examined. As shown in Fig. 6A, TRIM3 expression was upregulated in tamoxifen-resistant breast cancer compared with that in tamoxifen-sensitive breast cancer, and was strongly associated with ESR1 SUMOylation. Consistently, TRIM3 promoted the transcription of ER-targeted genes in tamoxifenresistant $\mathrm{ER}^{+}$breast cancer (Fig. 6B). Collectively, these results support the view that TRIM3 upregulation promotes ESR1 SUMO modification and ER signaling pathway activity, leading to tamoxifen resistance in $\mathrm{ER}^{+}$breast cancer.

\section{DISCUSSION}

Tamoxifen is a widely used treatment for patients with ER-positive breast cancer; however, de novo or acquired resistance results in failure of tamoxifen therapy, which is a clinical challenge $[4,5]$. In the present study, we found that TRIM3 was upregulated in tamoxifen-resistant breast cancer tissues compared with that tamoxifen-sensitive breast cancer tissues, and correlated closely with poorer survival in $\mathrm{ER}^{+}$breast cancer. Overexpression of TRIM3 contributed to cell survival upon tamoxifen treatment both in vivo and in vitro. Moreover, TRIM3 promoted the ESR1 SUMO modification and activated the transcription of ER-target genes by binding to UBC9, which could be abolished by overexpression of SENP1. Taken together, our results demonstrated that TRIM3 modifies the SUMOylation of ESR1 and confers tamoxifen resistance, which provided a novel therapeutic target for breast cancer therapy.

TRIM3, a member of RING-type E3 ubiquitin ligases, plays diverse roles in regulating neoplastic processes [21, 25-27]. TRIM3 was identified as a novel RING finger protein expressed in the rat brain, which is involved in regulating neuronal outgrowth $[19,28]$. TRIM3 is a candidate brain tumor suppressor gene, which suppresses brain tumorigenesis via attenuating Notch signaling and suppressing c-MYC expression [29, 30]. Furthermore, TRIM3 is downregulated in various types of cancer, 
A

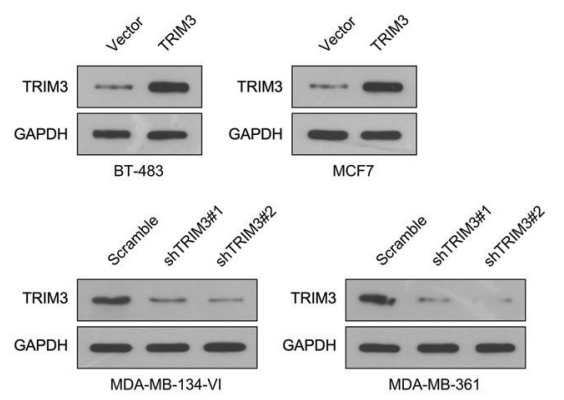

B

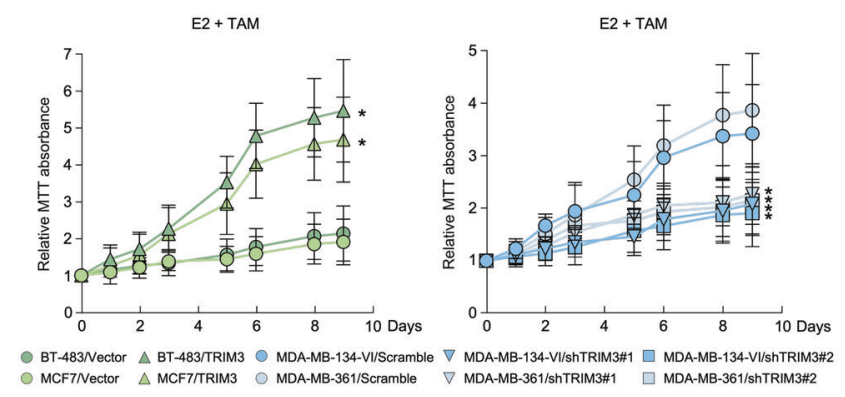

c
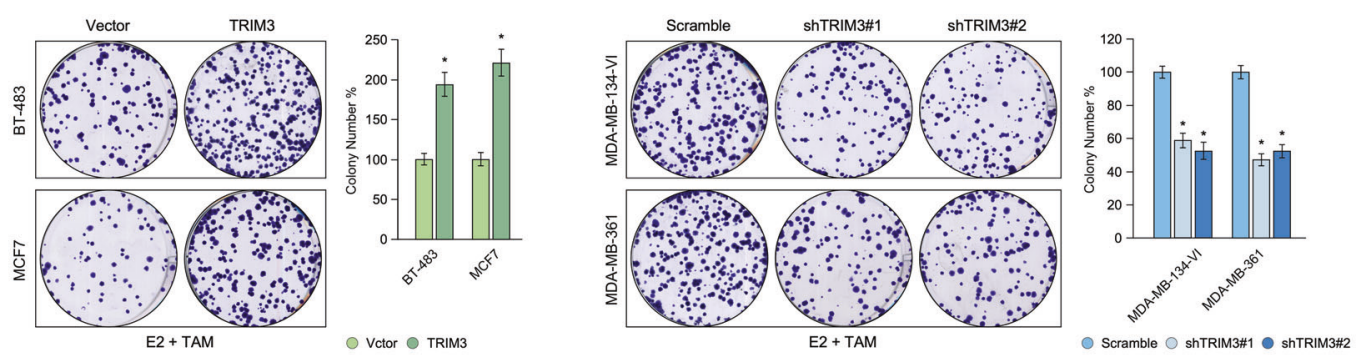

D
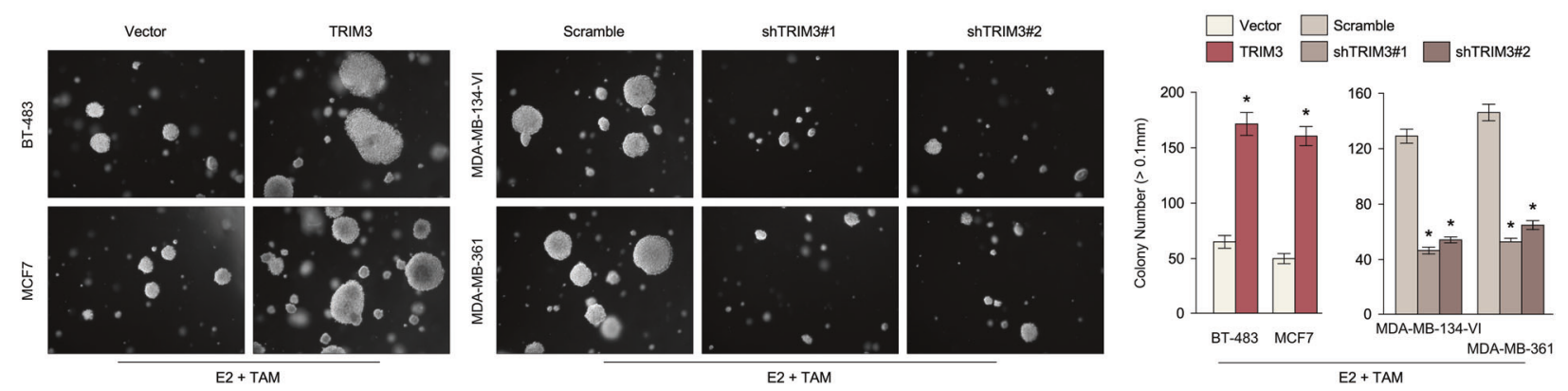

E
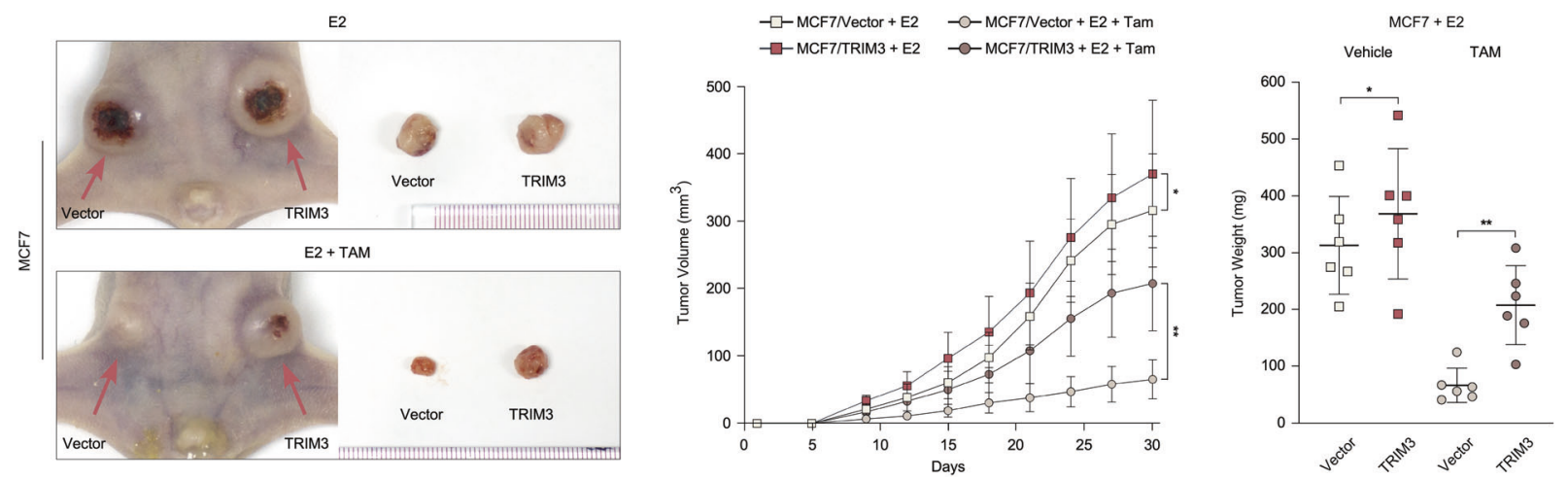

Fig. 2 TRIM3 contributes to tamoxifen resistance in breast cancer. A Western blotting analysis of TRIM3 levels in the indicated breast cancer cell lines stably overexpressing TRIM3 and silencing for TRIM3. Cell viability was measured using MTT (B) and colony formation (C) assays in the indicated cell lines treated with E2 $(10 \mathrm{nM})$ and TAM $(1 \mu \mathrm{M})$. Statistical data are presented as the mean \pm SD. D Soft agar assay of the indicated cells treated with E2 $(10 \mathrm{nM})$ and TAM $(1 \mu \mathrm{M})$ (left panel) and the statistical significance of colony formation was assessed in the right panel. Data are presented as the mean \pm SD. $P<0.05$. E Representative images of the tumors in the xenografts (left panel). Tumor growth curves (middle panel) and tumor weight (right panel) of the indicated xenograft tumors $(n=6 /$ group$)$. Data are presented as the mean \pm SD. ${ }^{*} P<0.05,{ }^{* *} P<0.01$

including liver cancer [20], esophageal squamous cell carcinoma [27], and colon cancer [31], and inhibits cell proliferation, invasion, and metastasis. However, the correlation between TRIM3 and tamoxifen resistance in breast cancer remains unclear.
In the current study, we found that TRIM3 showed significantly higher expression in recurrent $\mathrm{ER}^{+}$breast cancer than that in non-recurrent $\mathrm{ER}^{+}$breast cancer, which consistent with the analysis of TRIM3 expression in response to tamoxifen treatment 
A
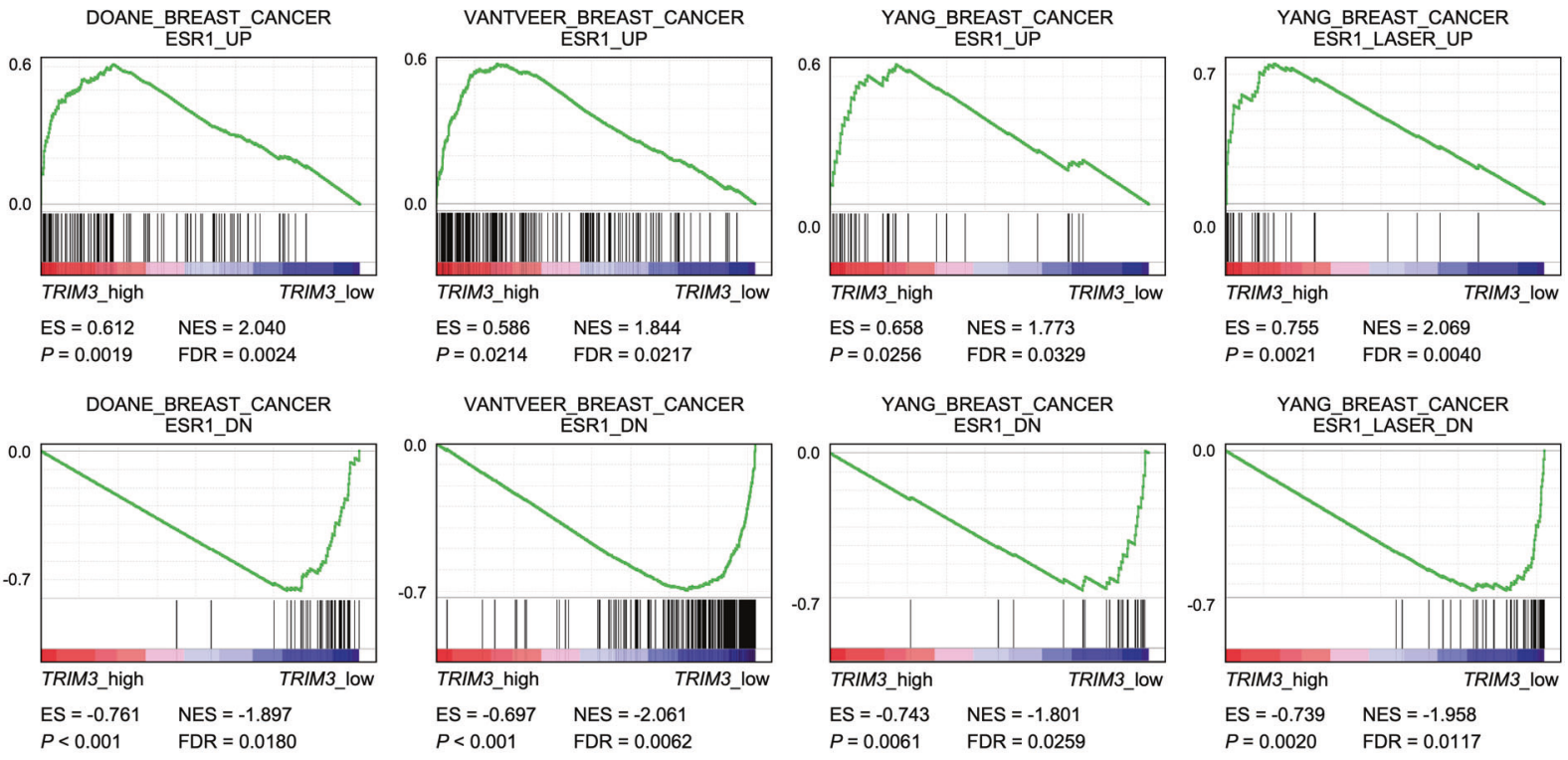

(TCGA ER positive breast cancer specimens, $n=400$ )

B
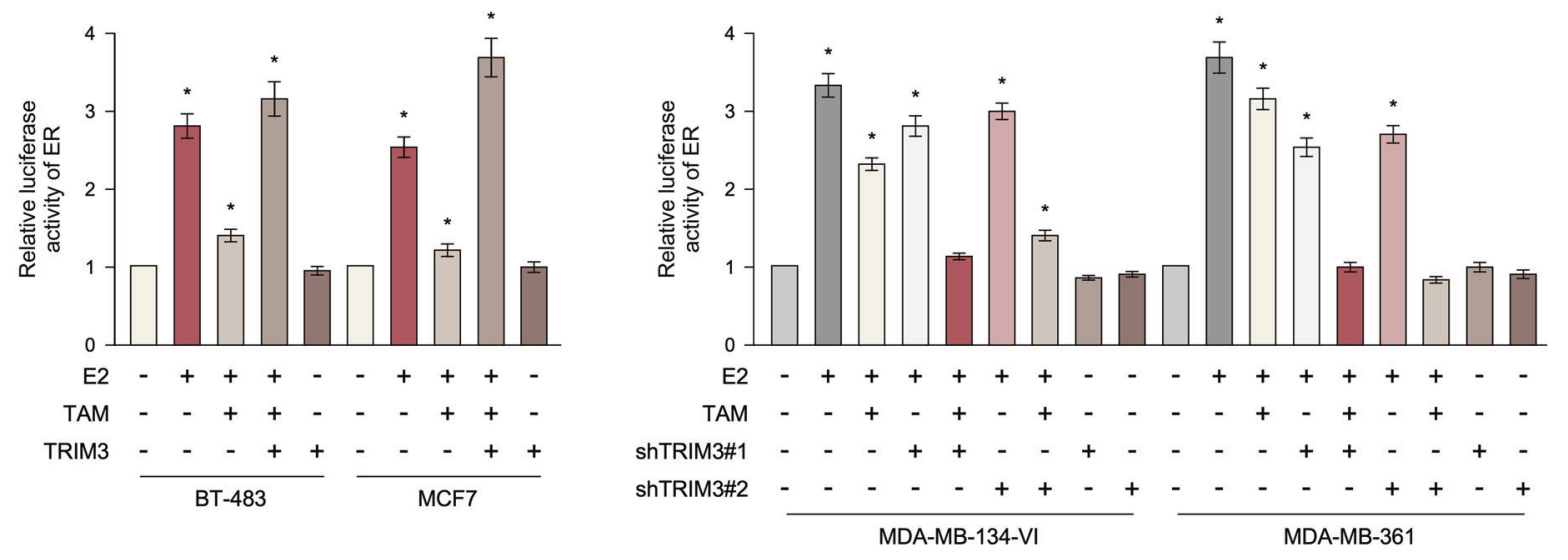

C

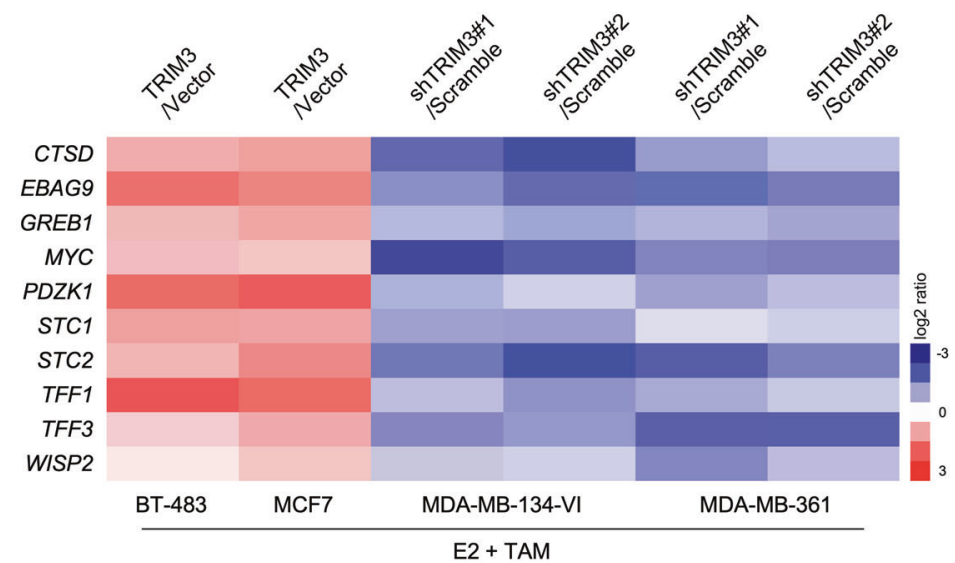

Fig. 3 TRIM3-induced estrogen receptor (ER)-dependent transcription in response to tamoxifen therapy. A GSEA showed that TRIM3 expression correlated positively with ESR1-activated gene signatures and negatively with ESR1-suppressed gene signatures in ER ${ }^{+}$breast cancer profiles (TCGA, $n=400)$. B Estrogen response element (ERE) luciferase reporter activity was analyzed in the indicated cells treated with E2 $(10 \mathrm{nM})$ and/or TAM $(1 \mu \mathrm{M})$. Data are presented as the mean \pm SD. ${ }^{*} P<0.05$. C Heatmap of ER-regulated gene expression in the indicated cells treated with E2 and TAM. 


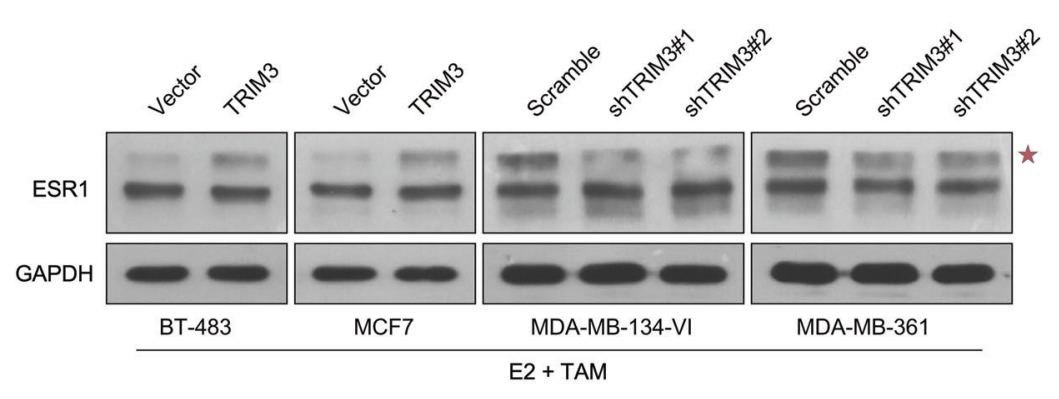

C

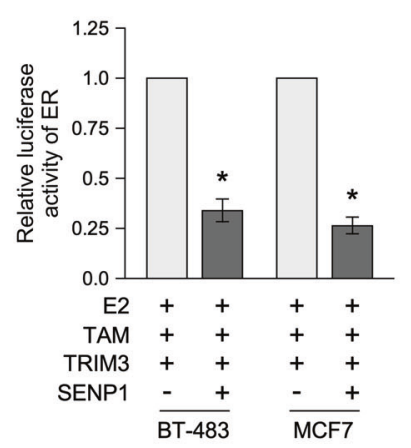

F
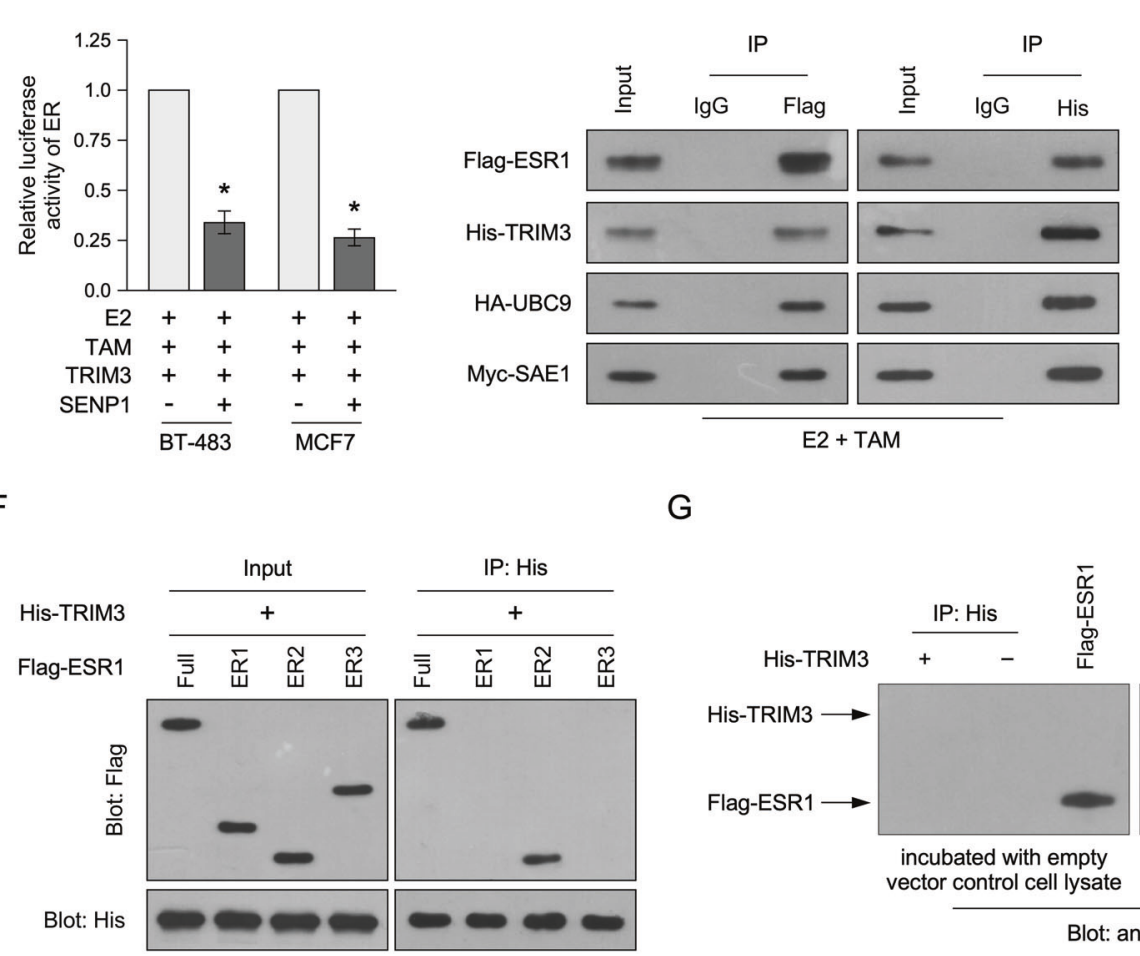

G

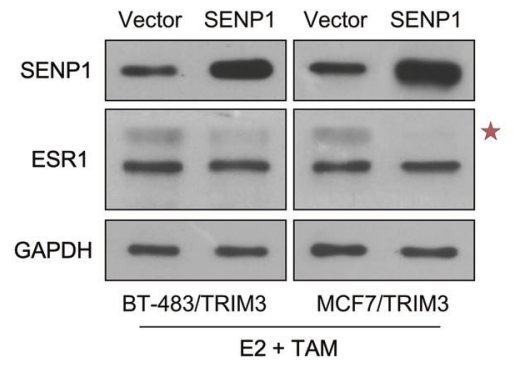

E
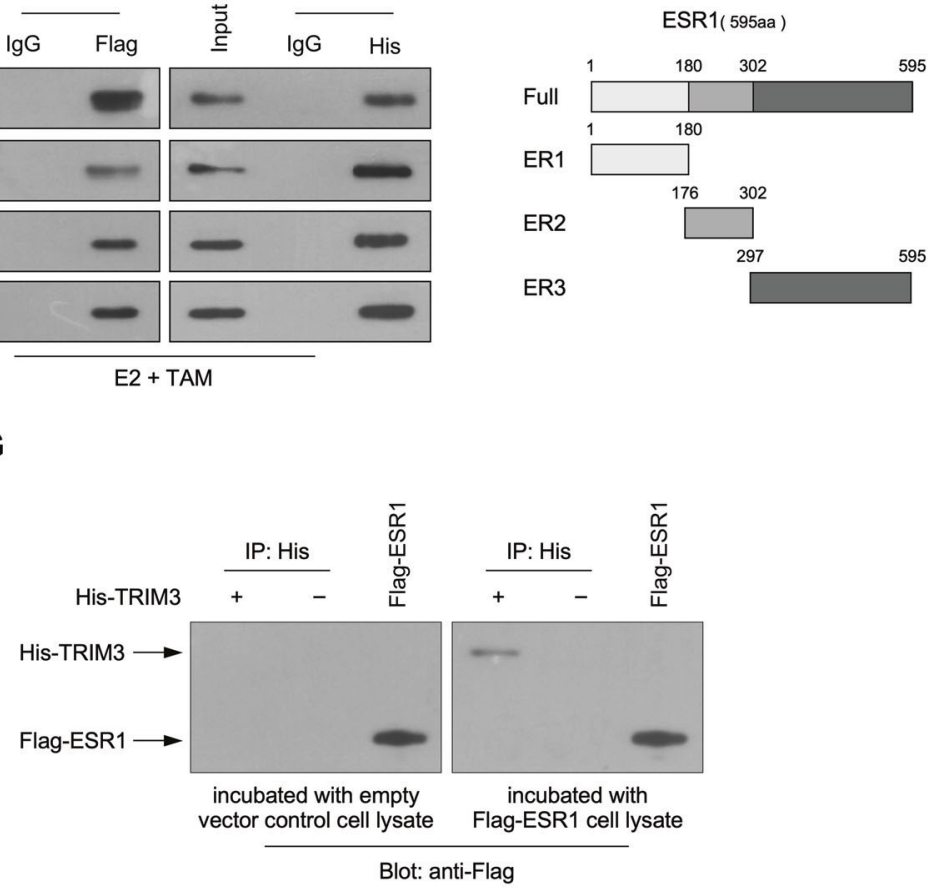

Fig. 4 TRIM3-induced SUMOylation of ESR1 via binding with UBC9. Western blotting of ESR1 (A, B) and SENP1 (B) levels in the indicated breast cancer cell lines. GAPDH was used as the loading control. A star represents SUMOylation of ESR1. C Estrogen response element (ERE) luciferase reporter activity was analyzed in the indicated cells treated with E2 (10 nM) and/or TAM $(1 \mu M)$. Data are presented as the mean \pm SD. ${ }^{*} P<0.05$. D Immunoprecipitation assay revealing that TRIM3 interacts with ESR1, UBC9, and SAE1 in the indicated cells. E Schematic illustration of wild-type ESR1 and truncated ESR1. F Immunoprecipitation assay revealing that TRIM3 interacts with the ER2 domain of ESR1. G Far-western blotting revealing that TRIM3 directly interacts with Flag-ESR1 in the cell lysate.

in $\mathrm{ER}^{+}$breast cancer data obtained from the TCGA (Figs. 1A, B and $6 \mathrm{~A}$ ). Furthermore, the expression of TRIM3 correlated with poorer OS, RFS, and DMFS in patients with $\mathrm{ER}^{+}$breast cancer upon tamoxifen treatment, but not in patients with $\mathrm{ER}^{-}$breast cancer (Figs. 1C and S1C), suggesting that TRIM3 might be a potential predictive marker for the response to tamoxifen in patients with $\mathrm{ER}^{+}$breast cancer.

ERa has been recognized as a favorable prognostic biomarker and has a determinative role for breast cancer therapy [32]. ERa belongs to the nuclear receptor superfamily, and activates the transcription of targeted genes to resist tamoxifen treatment $[33,34]$. SUMOylation, as a PTM, is hyperactivated in breast cancer and correlates with ERa signaling pathways [35]. Although SUMO modification represses transcription via binding with corepressors, including HDACs and DAXX [36, 37], growing evidence has shown that SUMOylation is involved in promoting transcriptional activity. For example, the transcriptional activity of ERa is upregulated in breast cancer via binding with SUMOylation proteins, such as CLOCK [22] and ZFP282 [38]. Furthermore, ERa was proven to be SUMOylated via binding with UBC9, and blocking its SUMOylation impaired the transcriptional activity of ERa through decreased DNA binding, without influencing the cellular localization of ERa [16]. Therefore, the precise molecular mechanism by which SUMO modification enhances the transcriptional activity of ERa in breast cancer requires further investigation.

In the present study, we identified that TRIM3 plays an important role in regulating the ER signaling pathway and confers tamoxifen resistance in breast cancer. Overexpression of TRIM3 enhanced ERE luciferase reporter activity and upregulated ERregulated genes upon tamoxifen treatment, whereas silencing of TRIM3 had the opposite effect (Fig. 3B, C). Furthermore, TRIM proteins regulate SUMO modification by transferring SUMO1 from UBC9 to the substrate $[17,18]$. Consistently, the SUMOylation of ESR1 was upregulated in TRIM3-transduced cells upon tamoxifen treatment, which was abolished by overexpression of SENP1 
A
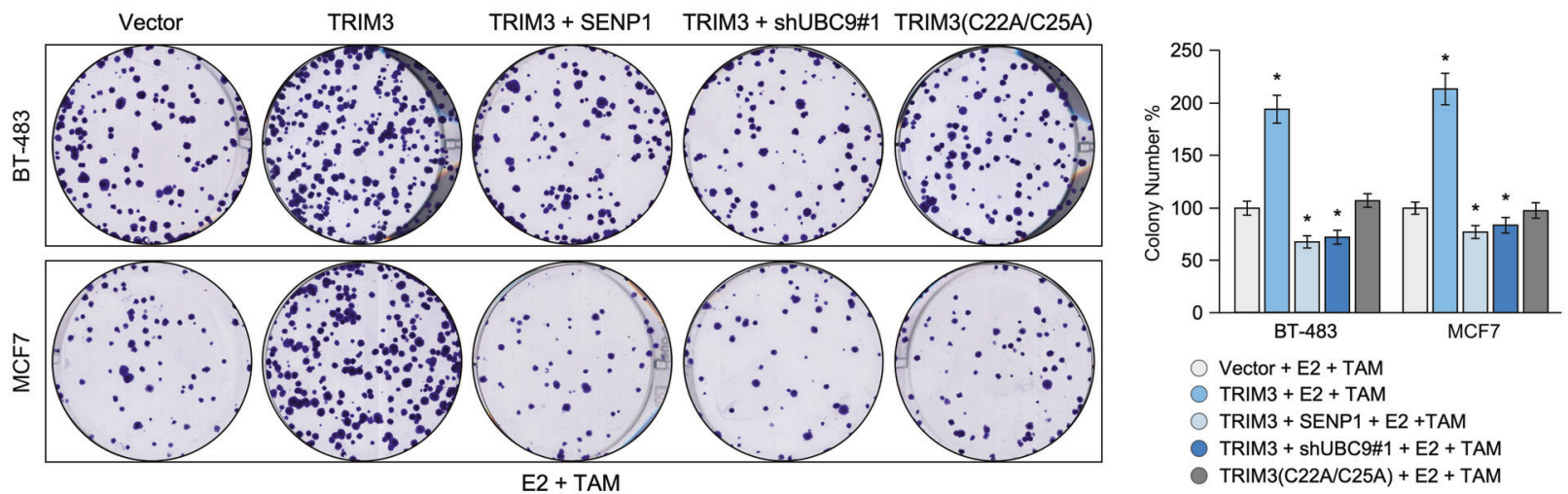

B
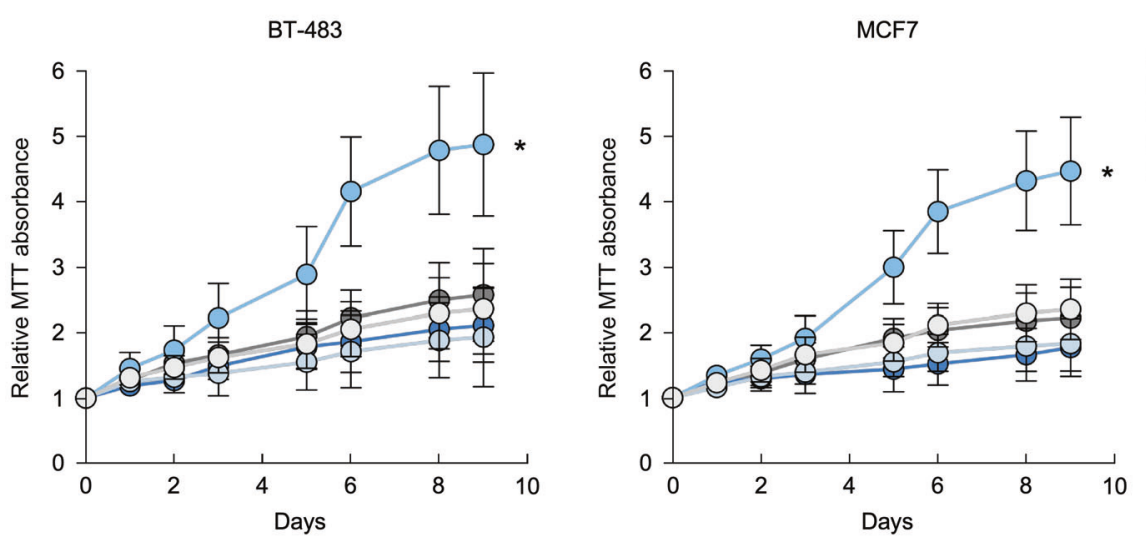

Vector + E2 + TAM

TRIM $3+\mathrm{E} 2+\mathrm{TAM}$

TRIM3 + SENP1 + E2 + TAM

TRIM3 + ShUBC9\#1 + E2 + TAM

TRIM3 $(C 22 A / C 25 A)+E 2+T A M$

C
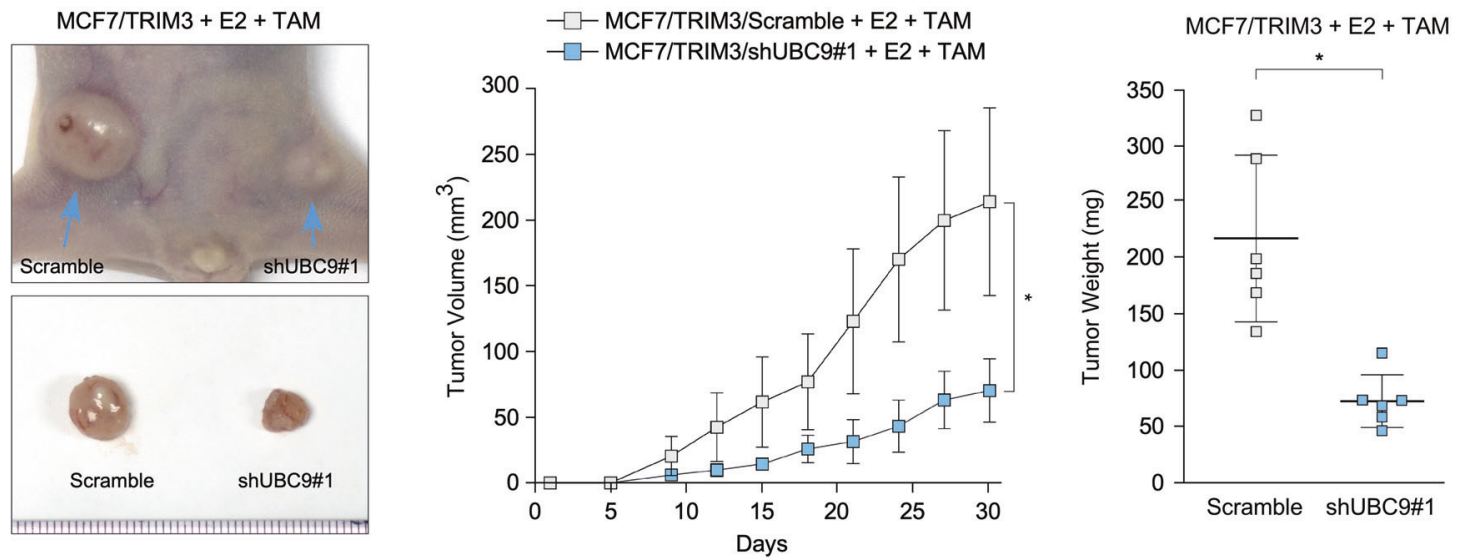

Fig. 5 Silencing UBC9 reverses the tamoxifen resistance effect of TRIM3. Representative images of colony formation (A) and MTT assays (B) of the indicated cells treated with E2 $(10 \mathrm{nM})$ and TAM $(1 \mu \mathrm{M})$. Histograms showing colonies formed by the indicated cells. Data are presented as the mean \pm SD. ${ }^{*} P<0.05$. C Representative images of the tumors in the xenografts (left panel), tumor growth curves (middle panel) and tumor weight (right panel) of the indicated xenograft tumors $\left(n=6 /\right.$ group). Data are presented as the mean \pm SD. ${ }^{*} P<0.05$.

(Fig. 4A, C). Furthermore, immunoprecipitation analysis demonstrated that TRIM3 interacted with ESR1, UBC9, and SAE1, suggesting that TRIM3 acts as a SUMO E3 ligase to transfer SUMO from SAE1 (E1) and UBC9 (E2) to ESR1 (substrate) (Fig. 4D). Importantly, TRIM3 interacted with ESR1 by binding directly to its DNA-binding domain (Fig. 4E-G), which might induce ESR1 transcriptional activity. Overexpression of UBC9 promoted breast cancer tumorigenesis, while a dominant negative mutant of UBC9 decreased tumorigenesis [39]. Similarly, blocking UBC9 expression also decreased cell survival and tumorigenesis in TRIM3-transduced cells upon E2 and tamoxifen treatment in vitro and in vivo (Fig. 5). To further examine the effect of catalytic dead mutant of TRIM3 in tamoxifen response in $\mathrm{ER}^{+}$breast cancer, we constructed TRIM3 mutant $(C 22 A / C 25 A)$, which affects its $E 3$ ligase activity $[2,3]$. Consistently, TRIM3 (C22A/C25A) rescued tamoxifen sensitivity in vitro and in vivo (Fig. 5A, B, Fig. S6). Therefore, these results suggest that regulation of TRIM3 tamoxifen resistance was dependent on its catalytic activity.

In conclusion, our results confirmed that TRIM3 acts as a SUMO E3 ligase to regulate ESR1 SUMO modification and transcriptional 
A

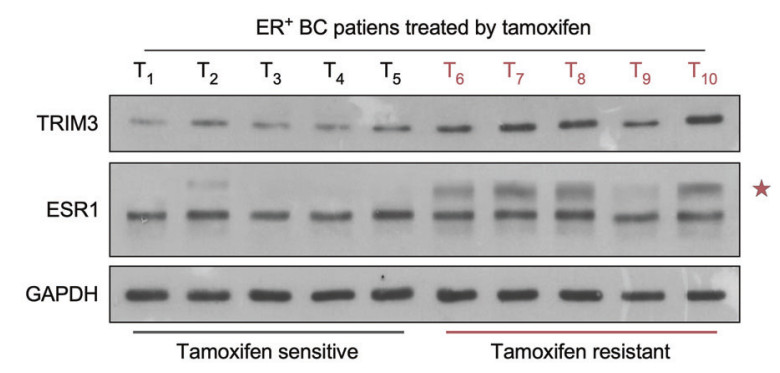

B

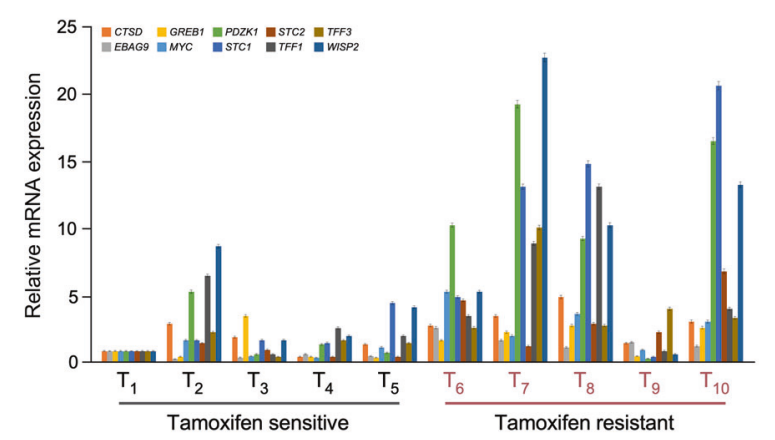

Fig. 6 TRIM3 promotes transcription of ER-target genes in clinical breast cancer. Western blotting of TRIM3 and ESR1 (A), qPCR of ERtargeted genes (B) in 10 cases of freshly $\mathrm{ER}^{+}$breast cancer treated with tamoxifen therapy, including tamoxifen-resistant and tamoxifen-sensitive tissues. GAPDH was used as the loading control. A star represents SUMOylation of ESR1.

activity, thus conferring tamoxifen resistance via the TRIM3/UBC9/ ESR1 axis in $\mathrm{ER}^{+}$breast cancer. Silencing UBC9 in TRIM3-transduced cells conferred tamoxifen sensitivity in breast cancer. Importantly, TRIM3 overexpression correlated with poor survival of patients with $\mathrm{ER}^{+}$breast cancer with tamoxifen resistance, identifying TRIM3 as a potential biomarker for the treatment of $\mathrm{ER}^{+}$breast cancer.

\section{MATERIALS AND METHODS}

\section{Cell culture}

The human breast cancer cell lines BT-474, BT-483, CAMA-1, HCC1428, HCC1500, MCF7, MDA-MB-134-VI, MDA-MB-175-VII, MDA-MB-361, T-47D, ZR75-1 ZR-75-30, and MCF/TR were cultured according to the manufacturer's instruction. Short tandem repeat (STR) profiling were used and authenticated in all cell lines. Mycoplasma eradication was evaluated by PCR.

\section{Chemical reagents}

$17 \beta$-estradiol (E2) and 4-hydroxytamoxifen (TAM) were purchased from Sigma-Aldrich (St. Louis, MO, USA).

\section{Tissue specimens and immunohistochemistry}

A cohort of paraffin-embedded breast cancer (48 cases) which received tamoxifen therapy was used to detect the expression of TRIM3 via AntiTRIM3 (ab111840). The specimens were obtained from the First Affiliated Hospital of Sun Yat-sen University between 2010 and 2015. 10 freshly breast cancer tissues with tamoxifen-sensitive and tamoxifen resistant were collected. Prior patient consent and approval were obtained.

\section{Constructs and transfection}

Genomic DNA of TRIM3 was PCR-amplified and cloned into a pBABE-puro retroviral vector. TRIM3 (C22A/C25A) was generated and cloned into a $\mathrm{pBABE}$-puro retroviral vector. The cloned into a $\mathrm{pBABE}$-puro retroviral vector. e pSUPER-puro shRNA of TRIM3 and UBC9 were purchased from Transheep Bio. All clone primer and siRNA oligonucleotides are listed as Table S2. Estrogen Response Element (ERE) was cloned into a pGL3 basic vector (Promega). The Renilla luciferase TK was used as transfection control. All cells overexpressing TRIM3 or silencing TRIM3 were selected with $0.5 \mu \mathrm{g} / \mathrm{ml}$ puromycin.

\section{Immunoprecipitation analysis}

Immunoprecipitation assay was performed according to described previously [40]. Lysates were incubated with Flag or His affinity beads (Sigma-Aldrich). The agarose beads were washed with wash buffer. Then the eluations were detected using appropriate antibodies.

\section{Far-western blotting}

Far immunoblotting was performed by using the proteins immunoprecipitated by anti-His antibody. The proteins were detected using western blotting. Empty vector or Flag-ESR1 cell lysate was added to the PVDF membrane and incubated overnight. Then the membrane was subjected to immunoblotting analysis by indicated antibody.

\section{Western blotting analysis}

Western blotting was performed using antibodies against TRIM3 (ab111840), ESR1 (ab108398), UBC9 (ab75854), SENP1 (ab108981) and SAE1 (ab185552). The membranes were re-probed with an anti-GAPDH (BOSTER, BM3876) as the loading control.

\section{Quantitative real-time reverse transcription PCR (qPCR)}

Total RNA was isolated with TRIzol reagent (Invitrogen) according to manufacturer's instructions. RNA was reverse-transcribed into CDNA and carried out via Real-time PCR with SYBR Green Master (Roche). The data were assessed base on the threshold cycle $(\mathrm{Ct})$, and calculated as $2^{-[(C}{ }_{t}$ of gene) - $\left(C_{t}\right.$ of GAPDH)] , which was normalized to GAPDH expression. All primers are listed as Table S2.

\section{Cell viability assay}

Indicated cells were treated with Estrogen and/or tamoxifen for $48 \mathrm{~h}$. The cells were added MTT dye (Sigma-Aldrich), and then the cells were resuspended with dimethyl sulfoxide (Sigma-Aldrich) and measured with automatic microplate reader.

\section{Colony formation assay}

Indicated cells were incubated at a level of $5 \% \mathrm{CO}_{2}$ at a temperature of $37^{\circ} \mathrm{C}$ for 2 weeks. Then the cells were fixed, stained with crystal violet stain, and counted.

\section{Luciferase activity assay}

Luciferase reporter plasmid and pRL-TK Renilla plasmid were transfected into indicated cells. After $48 \mathrm{~h}$, the cells were lysis and measured using a Dual Luciferase Reporter Assay (Promega) according to the manufacturer's instructions.

\section{Anchorage-independent growth ability assay}

Indicated cells were suspended with medium plus $0.33 \%$ agar, and then plated on top of $0.66 \%$ agar medium mix. After 10 days, colonies $>0.1 \mathrm{~mm}$ in diameter were counted. The experiments were performed in triplicates.

\section{Tumor xenografts}

Under the guideline of National Institutes of Health Guide for Care and Use of Laboratory Animals, xenografts were performed using athymic nude female mice (4-5 weeks of age, $18-20 \mathrm{~g}) .5 \times 10^{6}$ of indicated cells were injected into the left and right dorsal flank of mice implanted with E2 pellets $(0.72 \mathrm{mg} /$ pellet; 60-day release). After 1 week, a subcutaneous injection with or without tamoxifen pellet ( $5 \mathrm{mg} /$ pellet; 60 -day release). Tumor were examined twice a week, and tumor volume was calculated as $\left(L \times W^{2}\right) / 2$.

\section{Data processing and visualization}

The dataset is available in TCGA (https://tcga-data.nci.nih.gov/tcga/). Gene set enrichment analysis (GSEA) was performed on GSEA 2.0. 9 (http://www. broadinstitute.org/gsea/). The relationship between the expression of TRIM3 and ESR1 was determined by correlation coefficient. For the relationship between TRIM3 and the OS, RFS and DMFS of breast cancers, Kaplan-Meier Plotter (http://kmplot.com/analysis) was used. 


\section{Statistics'}

Unpaired Student's test was used to evaluate the statistical significance of the differences between two groups. Spearman's correlation analysis and Chisquare were used to evaluate the correlations. All $P$ values were two-tailed, and a value of $P<0.05$ was considered statistically significant. All data analyze were performed with SPSS19.0 software and presented with GraphPad Prism 8.0.

\section{REFERENCES}

1. Bray F, Ferlay J, Soerjomataram I, Siegel RL, Torre LA, Jemal A. Global cancer statistics 2018: GLOBOCAN estimates of incidence and mortality worldwide for 36 cancers in 185 countries. CA Cancer J Clin. 2018;68:394-424.

2. Musgrove EA, Sutherland RL. Biological determinants of endocrine resistance in breast cancer. Nat Rev Cancer. 2009;9:631-43.

3. Swaby RF, Sharma CG, Jordan VC. SERMs for the treatment and prevention of breast cancer. Rev Endocr Metab Disord. 2007;8:229-39.

4. Belachew EB, Sewasew DT. Molecular mechanisms of endocrine resistance in estrogen-positive breast cancer. Front Endocrinol. 2021;12:599586.

5. Clarke R, Liu MC, Bouker KB, Gu Z, Lee RY, Zhu Y, et al. Antiestrogen resistance in breast cancer and the role of estrogen receptor signaling. Oncogene. 2003;22:7316-39.

6. Berry DA, Muss HB, Thor AD, Dressler L, Liu ET, Broadwater G, et al. HER-2/neu and p53 expression versus tamoxifen resistance in estrogen receptor-positive, nodepositive breast cancer. J Clin Oncol. 2000;18:3471-9.

7. Parisot JP, Hu XF, DeLuise M, Zalcberg JR. Altered expression of the IGF-1 receptor in a tamoxifen-resistant human breast cancer cell line. Br J Cancer. 1999;79:693-700.

8. Ojo $D$, Wei $F$, Liu $Y$, Wang $E$, Zhang $H$, Lin $X$, et al. Factors promoting tamoxifen resistance in breast cancer via stimulating breast cancer stem cell expansion. Curr Med Chem. 2015;22:2360-74.

9. Pietras RJ, Arboleda J, Reese DM, Wongvipat N, Pegram MD, Ramos L, et al. HER-2 tyrosine kinase pathway targets estrogen receptor and promotes hormoneindependent growth in human breast cancer cells. Oncogene. 1995;10:2435-46.

10. Jeselsohn R, Buchwalter G, De Angelis C, Brown M, Schiff R. ESR1 mutations-a mechanism for acquired endocrine resistance in breast cancer. Nat Rev Clin Oncol. 2015;12:573-83.

11. Kim C, Tang G, Pogue-Geile KL, Costantino JP, Baehner FL, Baker J, et al. Estrogen receptor (ESR1) mRNA expression and benefit from tamoxifen in the treatment and prevention of estrogen receptor-positive breast cancer. J Clin Oncol. 2011;29:4160-7.

12. Giacinti L, Claudio PP, Lopez M, Giordano A. Epigenetic information and estrogen receptor alpha expression in breast cancer. Oncologist. 2006;11:1-8.

13. Yang $X$, Phillips DL, Ferguson AT, Nelson WG, Herman JG, Davidson NE. Synergistic activation of functional estrogen receptor (ER)-alpha by DNA methyltransferase and histone deacetylase inhibition in human ER-alpha-negative breast cancer cells. Cancer Res. 2001;61:7025-9.

14. Hartmaier RJ, Trabucco SE, Priedigkeit N, Chung JH, Parachoniak CA, Vanden Borre $\mathrm{P}$, et al. Recurrent hyperactive ESR1 fusion proteins in endocrine therapyresistant breast cancer. Ann Oncol. 2018;29:872-80.

15. Zhang X, Tanaka K, Yan J, Li J, Peng D, Jiang Y, et al. Regulation of estrogen receptor alpha by histone methyltransferase SMYD2-mediated protein methylation. Proc Natl Acad Sci USA. 2013;110:17284-9.

16. Sentis S, Le Romancer M, Bianchin C, Rostan MC, Corbo L. Sumoylation of the estrogen receptor alpha hinge region regulates its transcriptional activity. Mol Endocrinol. 2005;19:2671-84.

17. Hatakeyama S. TRIM proteins and cancer. Nat Rev Cancer. 2011;11:792-804.

18. Chu Y, Yang X. SUMO E3 ligase activity of TRIM proteins. Oncogene. 2011;30:1108-16.

19. El-Husseini $A E$, Fretier $P$, Vincent $S R$. Cloning and characterization of a gene (RNF22) encoding a novel brain expressed ring finger protein (BERP) that maps to human chromosome 11p15.5. Genomics. 2001;71:363-7.

20. Huang XQ, Zhang XF, Xia JH, Chao J, Pan QZ, Zhao JJ, et al. Tripartite motifcontaining 3 (TRIM3) inhibits tumor growth and metastasis of liver cancer. Chin Cancer. 2017;36:77.

21. Liu Y, Raheja R, Yeh N, Ciznadija D, Pedraza AM, Ozawa T, et al. TRIM3, a tumor suppressor linked to regulation of p21(Waf1/Cip1.). Oncogene. 2014;33:308-15.

22. Li S, Wang M, Ao X, Chang AK, Yang C, Zhao F, et al. CLOCK is a substrate of SUMO and sumoylation of CLOCK upregulates the transcriptional activity of estrogen receptor-alpha. Oncogene. 2013;32:4883-91.

23. Traboulsi T, El Ezzy M, Dumeaux V, Audemard E, Mader S. Role of SUMOylation in differential ERalpha transcriptional repression by tamoxifen and fulvestrant in breast cancer cells. Oncogene. 2019;38:1019-37.

24. Kobayashi S, Shibata H, Yokota K, Suda N, Murai A, Kurihara I, et al. FHL2, UBC9, and PIAS1 are novel estrogen receptor alpha-interacting proteins. Endocr Res. 2004;30:617-21.

25. Fu H, Yang $H$, Zhang $X$, Wang B, Mao J, Li X, et al. Exosomal TRIM3 is a novel marker and therapy target for gastric cancer. J Exp Clin Cancer Res. 2018;37:162.
26. Raheja R, Liu Y, Hukkelhoven E, Yeh N, Koff A. The ability of TRIM3 to induce growth arrest depends on RING-dependent E3 ligase activity. Biochem J. 2014;458:537-45.

27. Zhu J, Wu G, Ke Z, Cao L, Tang M, Li Z, et al. Targeting TRIM3 deletion-induced tumor-associated lymphangiogenesis prohibits lymphatic metastasis in esophageal squamous cell carcinoma. Oncogene. 2019;38:2736-49.

28. van Diepen MT, Spencer GE, van Minnen J, Gouwenberg Y, Bouwman J, Smit AB, et al. The molluscan RING-finger protein L-TRIM is essential for neuronal outgrowth. Mol Cell Neurosci. 2005;29:74-81.

29. Mukherjee S, Tucker-Burden C, Zhang C, Moberg K, Read R, Hadjipanayis C, et al. Drosophila Brat and Human Ortholog TRIM3 Maintain Stem Cell Equilibrium and Suppress Brain Tumorigenesis by Attenuating Notch Nuclear Transport. Cancer Res. 2016;76:2443-52.

30. Chen G, Kong J, Tucker-Burden C, Anand M, Rong Y, Rahman F, et al. Human Brat ortholog TRIM3 is a tumor suppressor that regulates asymmetric cell division in glioblastoma. Cancer Res. 2014;74:4536-48.

31. Piao MY, Cao HL, He NN, Xu MQ, Dong WX, Wang WQ, et al. Potential role of TRIM3 as a novel tumour suppressor in colorectal cancer (CRC) development. Scand J Gastroenterol. 2016;51:572-82.

32. Jensen EV, Jordan VC. The estrogen receptor: a model for molecular medicine. Clin Cancer Res. 2003;9:1980-9.

33. Gao A, Sun T, Ma G, Cao J, Hu Q, Chen L, et al. LEM4 confers tamoxifen resistance to breast cancer cells by activating cyclin D-CDK4/6-Rb and ERalpha pathway. Nat Commun. 2018;9:4180.

34. He H, Sinha I, Fan R, Haldosen LA, Yan F, Zhao C, et al. C-Jun/AP-1 overexpression reprograms ERalpha signaling related to tamoxifen response in ERalpha-positive breast cancer. Oncogene. 2018;37:2586-2600.

35. Karamouzis MV, Konstantinopoulos PA, Badra FA, Papavassiliou AG. SUMO and estrogen receptors in breast cancer. Breast Cancer Res Treat. 2008;107:195-210.

36. Girdwood D, Bumpass D, Vaughan OA, Thain A, Anderson LA, Snowden AW, et al. P300 transcriptional repression is mediated by SUMO modification. Mol Cell. 2003;11:1043-54.

37. Kuo HY, Chang CC, Jeng JC, Hu HM, Lin DY, Maul GG, et al. SUMO modification negatively modulates the transcriptional activity of CREB-binding protein via the recruitment of Daxx. Proc Natl Acad Sci USA. 2005;102:16973-8.

38. Yu EJ, Kim SH, Kim MJ, Seo WY, Song KA, Kang MS, et al. SUMOylation of ZFP282 potentiates its positive effect on estrogen signaling in breast tumorigenesis. Oncogene. 2013;32:4160-8.

39. Mo YY, Yu Y, Theodosiou E, Ee PL, Beck WT. A role for Ubc9 in tumorigenesis. Oncogene. 2005;24:2677-83.

40. Lin JS, Lai EM. Protein-protein interactions: co-immunoprecipitation. Methods Mol Biol. 2017;1615:211-9.

\section{ACKNOWLEDGEMENTS}

This study was supported by the National Natural Science Foundation of China [grant numbers 81772800 and 82072945$]$.

\section{AUTHOR CONTRIBUTIONS}

$\mathrm{RY}, \mathrm{NA}$, and XK designed this study, analyzed data and wrote the manuscript. $\mathrm{HZ}$ and NS performed the vitro experiments. $R Y$ and $Y L$ performed the vivo experiments. $R Y$ and XK contributed to collection of clinical samples. PL and SW provided important comments for the manuscript. All authors read and approved the final manuscript.

\section{COMPETING INTERESTS}

The authors declare no competing interests.

\section{ADDITIONAL INFORMATION}

Supplementary information The online version contains supplementary material available at https://doi.org/10.1038/s41389-021-00350-x.

Correspondence and requests for materials should be addressed to Runyi Ye, Pian Liu or Shenming Wang.

Reprints and permission information is available at http://www.nature.com/ reprints

Publisher's note Springer Nature remains neutral with regard to jurisdictional claims in published maps and institutional affiliations. 
(c) (i) Open Access This article is licensed under a Creative Commons Attribution 4.0 International License, which permits use, sharing, adaptation, distribution and reproduction in any medium or format, as long as you give appropriate credit to the original author(s) and the source, provide a link to the Creative Commons license, and indicate if changes were made. The images or other third party material in this article are included in the article's Creative Commons license, unless indicated otherwise in a credit line to the material. If material is not included in the article's Creative Commons license and your intended use is not permitted by statutory regulation or exceeds the permitted use, you will need to obtain permission directly from the copyright holder. To view a copy of this license, visit http://creativecommons. org/licenses/by/4.0/.

(c) The Author(s) 2021 